\title{
Laminin-incorporated nerve conduits made by plasma treatment for repairing spinal cord injury
}

\author{
Henrich Cheng ${ }^{\text {a }}$, Yi-Cheng Huang ${ }^{\text {b }}$, Pei-Teh Chang ${ }^{\text {a }}$, Yi-You Huang ${ }^{b, *}$ \\ ${ }^{a}$ Neurosurgery Neurological Institute, Taipei Veterans General Hospital, National Taiwan University, Taipei 10617, Taiwan \\ b Institute of Biomedical Engineering, College of Engineering, College of Medicine, National Taiwan University, Taipei 10617, Taiwan
}

Received 21 March 2007

Available online 18 April 2007

\begin{abstract}
To better direct the repair of damaged axons following spinal cord injury (SCI), we designed a nerve conduit (NC) modeled after the intact spinal cord, which would enable the axons to cross the lesioned area to rejoin on the other side. The NC consisted of a porous chitosan scaffold and was incorporated with laminin (LN) on the inner surface through oxygen plasma treatment. According to the $\mathrm{BBB}, \mathrm{CBS}$, and treadmill analyses, we found that following the implantation of the laminin-coated NC (LN-NC) the rats showed a tendency towards behavior improvement and functional recovery. Histology and immunocytochemical analyses indicated that the NC groups were capable of leading the damaged axons through the lesioned area without triggering inflammation or apoptosis. Together with the significantly enhanced expression of local GAP-43 in the LN-NC groups, as evidenced by western blot analysis, axon re-growth mediated by LN-NC was found to compare better than that by NC group. These results suggest a new possible approach to repairing SCI and, in general, a model which will be useful for other multidisciplinary procedures for complex neurological situations.
\end{abstract}

(C) 2007 Elsevier Inc. All rights reserved.

Keywords: Spinal cord injury (SCI); Chitosan; Oxygen plasma treatment; Laminin (LN); Nerve conduit (NC); Laminin-coated nerve conduit (LN-NC)

Tissue regeneration of peripheral nerves or the spinal cord following transection injury is a complex phenomenon. Once the nervous system is impaired, failures in other parts of the body may follow and it is very difficult to achieve functional recovery. To increase the prospects of axonal regeneration and functional recovery, numerous grafting procedures have been used, including implantation of autografts, allografts, and xenografts $[1,2]$. However, a number of problems may arise from these grafts. Instead, artificial nerve conduits were development as substitutes for nerve regeneration. Several particular characteristics should be considered for artificial nerve conduits, such as biocompatibility, biodegradability, lumen surface specialties and wall porosity, and permeability. In brief, nerve conduits with all the properties could become more "cell friendly" to promote nerve repair.

\footnotetext{
${ }^{*}$ Corresponding author. Fax: +886223940049.

E-mail addresses: d91548006@ntu.edu.tw (Y.-C. Huang), yyhuang@ ha.mc.ntu.edu.tw (Y.-Y. Huang).
}

We have devised a porous chitosan nerve conduit using a lyophilizing and wire-heating process [3]. Chitosan, the widely used biomaterial in our research, is not only biocompatible, biodegradable, low toxicity and cost but also has excellent potential for nerve repair [4-6]. Nevertheless, the key characteristics of chitosan are critical to its degree of deacetylation (DDA) value [7]. The DDA value of chitosan impacts the cell adhesion and neurite extension, which is important for tissue engineering in nervous system [8]. In this research, we adjusted the chitosan's DDA value by alkaline hydrolysis to control the amine content, and then made the conduit more suitable for nerve regeneration.

To make our chitosan NC more "cell friendly", we incorporated laminin (LN) into the inner surface of conduits by oxygen plasma treatment. This is achieved by placing the $\mathrm{NC}$ in contact with the gas, oxygen, in the treatment and imposing high energy radiation sufficient to ionize the gas to a plasma state. This method has been widely used for surface modification of biomaterials 
[9-12]. Using oxygen plasma treatment, we have successfully incorporated laminin on the surface of the chitosan membrane [13]. For further research of our experiments in this paper, we followed this same technique to incorporate laminin firmly on the inner surface of the porous chitosan NC. Furthermore, to preserve the microstructure of chitosan scaffold, oxygen plasma treatment time is critical. According to our results, the minimum plasma treatment time needed is $5 \mathrm{~min}$.

In this study, the LN-NC was tailored to fit into the cavity created by hemisection in the spinal cord of adult rats. If axons can traverse the injury site through the LN-NC, this is evidence that they may re-grow in unscarred regions. It means that the LN-NC we have devised might facilitate the process of nerve regeneration. We hypothesized that this laminin-modified, biodegradable, chitosan nerve conduit with specific architecture would impede glial scar formation, direct cell re-growth, facilitate axonal regeneration, and guide axon repair in the damaged spinal cord.

\section{Materials and methods}

Analysis of the degree of deacetylation (DDA)

Chitosan (DDA: $96.4 \pm 0.72 \%$ ) was synthesized by immersing commercially available chitosan (DDA: $82.5 \pm 1.15 \%$, Mw 645,000, Sigma Chemical Co.) flakes in a $40 \%$ aqueous solution of sodium hydroxide for $2 \mathrm{~h}$ at $110^{\circ} \mathrm{C}$. The DDA of chitosan samples was determined using ${ }^{1} \mathrm{H}$ NMR spectroscopy (Bruker AV400 MHz) according to a modified published procedure $[14,15]$. In brief, $10 \mathrm{mg}$ chitosan was added into a solution composed of $1.96 \mathrm{ml}$ Deuterium oxide $\left(\mathrm{D}_{2} \mathrm{O}, \mathrm{L}-4501\right.$, Sigma-Aldrich, USA) and $0.04 \mathrm{ml}$ deuterium chloride ( $\mathrm{DCl}, 35 \mathrm{wt} \%$ in $\mathrm{D}_{2} \mathrm{O}$, SigmaAldrich, USA) and waited about half an hour to ensure complete dissolution. The concentration of polymer in the solution was approximately $0.5 \%(\mathrm{w} / \mathrm{v})$. The DDA was calculated by comparing the integrated area of the signal group of $\mathrm{H} 1$ or $\mathrm{H} 2-\mathrm{H} 6$ with that of the signal of the methyl group, as previously described $[14,15]$.

\section{Fabrication of nerve conduits (NCs)}

The nerve conduits (NCs) were fabricated by lyophilization and a wireheating process [3]. Briefly, the chitosan/acetic acid solution $(2 \% \mathrm{w} / \mathrm{v})$ was injected into the mold with a Ni-Cr wire as a mandrel. Acetic acid $(98 \%$, Wako Pure Chemical Industries, Ltd.) was used as received. After the wire framework mold was frozen, the $\mathrm{Ni}-\mathrm{Cr}$ wire was heated by increasing the voltage of the power supply (Tai Yee Shing, Taiwan), and then pulled out from the scaffold as soon as possible, usually less than one minute. The acetic acid was then sublimated using temperature-controlled lyophilizers (VirTis, NY, USA). After lyophilization, the NCs were washed by 1:1 $0.1 \mathrm{M} \mathrm{NaOH}-\mathrm{MeOH}$ and $1: 1 \mathrm{MeOH}$-water in order to neutralize the acid, and then dried again by lyophilizers. Finally, the nerve conduits were stored in a dry environment until use.

\section{Incorporation of laminin into NCs}

To make the NCs hydrophilic and chemically active, they were first treated with oxygen plasma. The plasma treatment was performed between two parallel plate electrodes in a glow discharge quartz reactor (Model SP100 Plasma System, manufactured by Anatech Co., Ltd., USA), with power supply set at $50 \mathrm{~W}, 40 \mathrm{kHz}$. The NCs were placed on the ground electrode facing upward and exposed to the glow discharge at an oxygen pressure of $36 \mathrm{mTorr}$ for 5 or $10 \mathrm{~min}$ for the subsequent laminin (L-2020; Sigma, St. Louis, MO) coupling reaction. Then, $200 \mu \mathrm{l}$ of laminin solution $(100 \mu \mathrm{g} / \mathrm{ml})$ were added at $4{ }^{\circ} \mathrm{C}$ over $3 \mathrm{~h}$. Finally, the laminin-modified NCs were washed several times with PBS buffer.

\section{Characterization of laminin-modified NCs ( $L N-N C s)$}

The morphology of the LN-NCs was identified under light microscope (Olympus IX70, Japan). We applied the hematoxylin/eosin (H\&E) staining method to picture the scaffold more clearly. To visualize the distribution of proteins in the oxygen plasma-treated NCs, we prepared representative samples according to the methods described above, but using Rhodamine-BSA (red fluorescence) instead of laminin. After preparation, we rinsed the channels in PBS buffer and viewed the protein distribution using a confocal microscope (TCS SP2, Leica, Major Instruments Co., Ltd.) in fluorescence mode.

\section{Surgical procedures and animal care}

Adult $250 \mathrm{~g}$ female Sprague-Dawley rats were used in these studies. All procedures involving animals were approved by the Animals Committee of the Taipei Veterans General Hospital. The spinal cords of the SD rats were completely transected at $\mathrm{T} 8$ and a $5 \mathrm{~mm}$ piece of spinal cord tissue was removed. The $5 \mathrm{~mm}$ gap in the severed spinal cord was bridged by implantation of a LN-NC or NC conduit. At specified post-injury times, 1 or 2 months, the rats received an overdose of anesthetic sodium pentobarbital. For tissue section staining, we collected 1-cm-long samples of the spinal cord segment rostral to the injury site. The rats were perfused with saline followed by $4 \%$ buffered paraformaldehyde (PFA), and the spinal cords were post-fixed for $1 \mathrm{~h}$ in PFA and carried through sucrose gradients. The spinal cords were embedded in tissue freezing medium (OCT) Tissue Tek mounting media and cut with a cryostat. Using cryosection, we made $10 \mathrm{~mm}$ serial coronal specimens of the spinal cords and stored them at $-20^{\circ} \mathrm{C}$. The group of NC/LN-NC transplantation contains at less 3 animals $(n \geqslant 3)$, respectively.

\section{Behavior analysis}

$B B B$ and $C B S$ scores. To ensure that all experimental animals received similar degrees of injury and to monitor the rate of their recovery, the rats all underwent the Basso, Beattie and Bresnehan locomotion test (BBB) and the Combined Behavior Score (CBS) following their injury, to test for functional deficits $[16,17]$.

Robotic recording of locomotor functions. A robotic rat stepper commercialized by Robomedica, Inc. (Rodent Robot) was used in this study. The robots sampled the body weight support (BWS) levels and the positions of the rats' lower shanks and stored these measurements in digital form at $100 \mathrm{~Hz}$ on the robot control computer's storage drive. We conducted detailed evaluations of the rats' stepping ability after conduit transplantations in the SCI rats. At the recordings, the rats were allowed to step on the treadmill for $30 \mathrm{~s}$, at $75 \%$ BWS, without the robots attached. For each analysis, the SCI rats were evaluated on paw placement, weight bearing, and movement ability. The robot trajectories were segmented into individual steps by the step detection algorithm and then analyzed to determine step length, step height, and toe-off position, as well as step cycle, swing, and stance durations as a function of BWS [18].

\section{Immunohistochemistry}

The sections of spinal cord obtained by cryosection were incubated with the primary antibodies of interest, such as GAP-43, tubulin beta III, and ED-1. The sections were then washed in PBS, and incubated in secondary antibodies conjugated to fluorephore, or secondary antibodies conjugated to biotin, then streptavidin-conjugated fluorephore, to increase sensitivity. Alternatives to this procedure can be incubating the sections in Avidin-biotin-peroxidase complex (ABC) (Vector lab), following incubation of the primary antibody, and subsequently developing the peroxidase by incubating the sections in diaminobenzidin (DAB) solution in order to 
visualize the peroxidase label, or a kit from the Vector lab for chromogen development of desire.

\section{Immunoblots}

The animals were perfused with $4 \%$ PFA in phosphate buffer. The spinal cords were collected, immersed overnight in 4\% PFA in phosphate buffer and then transferred to a $30 \%$ sucrose solution. Horizontal or transverse sections of spinal cord, obtained by cryosection $(20-\mu \mathrm{m}$ thickness), were placed on poly-L-lysine-coated slides for immunostaining. Following the removal of ten slices from each rat, the spinal sections were incubated with $1 \mathrm{ml}$ of $50 \mathrm{mM} \mathrm{NH} \mathrm{Nl}_{4} \mathrm{Cl}$ aoom temperature (RT) for $10 \mathrm{~min}$, then washed with $1 \times \mathrm{PBS}$, and incubated with $1 \mathrm{ml}$ of $50 \mathrm{mM}$ Tris- $\mathrm{HCl} \mathrm{pH} 7.4$ at $\mathrm{RT}$ for $10 \mathrm{~min}$, then washed again. Protein samples were extracted in 1\% NP-40 lysis buffer and detected using Bradford protein assay. Equal amounts of protein were then loaded onto $3.5-12.5 \%$ gradient SDS-PAGE gel.

The electrophoresis was performed according to standard procedures; the proteins were transferred to a Polyvinylidene fluoride (PVDF) membrane, which was incubated with anti-GAP43 as the primary antibody. SuperSignal Chemiluminescent Substrate (Pierce, Rockford, IL) was used to select the applicable secondary antibody and to detect the horseradish peroxidase (HRP) on the immunoblot. The related optic densities of the GAP-43 positive bands were quantified using imaging, processing and analysis in Java ImageJ (released from NIH).

\section{Results and discussion}

\section{Characteristics of adjusted chitosan}

The $400 \mathrm{MHz}{ }^{1} \mathrm{H}$ NMR spectra of chitosan with different DDA value at $70^{\circ} \mathrm{C}$ were showed in Supplementary Fig. 1. Through alkaline hydrolysis, the DDA value of chitosan was successfully adjusted to $96.4 \pm 0.72 \%$. Controlling the degree of deacetylation can regulate the specific characteristics of chitosan scaffold. T. Freier et al. [7,19] showed that chitosan with higher deacetylation had a lower degradation rate, higher mechanical strength, higher cell compatibility and cell adhesion. They also found that chitosan with $99.5 \%$ and $89 \%$ deacetylation showed more and longer neurite outgrowth in vitro than the other samples studied, which demonstrated that nerve cell affinity with chitosan depended on the levels of amine in the chitosan. By adjusting its amine content, chitosan can be tuned for optimal biodegradation and cell compatibility, which are important for tissue engineering in the nervous system. The DDA value of chitosan used in this paper was therefore adjusted to $96.4 \pm 0.72 \%$.

\section{Laminin distribution in $\mathrm{NCS}$}

In order to determine the protein distribution within the $\mathrm{NC}$ prepared by plasma treatment method described in 'Incorporation of laminin into NCs', Rhodamine-BSA, instead of laminin, was loaded into the channel and viewed using fluorescent microscopy (Fig. 1A). The protein was mainly localized on the inner surface of the NC. Furthermore, a series of confocal microscopic pictures also showed that the protein appeared to be distributed throughout the inner surface of the entire channel wall (data not shown). Laminin localized in the inner portion of the channel wall should have facilitated nerve regeneration, since laminin assists Schwann cells adhesion and then guides neurite growth. Although the exact underlying mechanisms regulating dynamic axon/schwann cell apposition are unknown, experiments support the concept that schwann cells offer highly preferred substrate for axon migration and release bioactive factors that further enhance nerve migration [20-22]. In vitro, laminin-coated substrate has been proven to enhance schwann cell attachment and affinity indeed [13]. We infer that, in vivo, the LN-NCs could lead axon regeneration within the channel and enhance regenerative capacity.

Compared with conventional chemical method for material surface modification, plasma treatment is the only way to incorporated laminin on the inner surface of NCs. Oxygen plasma goes through the channel of conduits directly, collide with the inner surface of the conduits, and then activate the chitosan chain of the surface. The laminin added could react with the free radical formed on the inner surface. As a result, modification can be confined to the surface layer without modifying the bulk properties of the polymer [23]. For conventional chemical method, surface modification will be formed for the whole structure because the chemical solution will penetrate the whole conduit instead of confining to the inner surface. Therefore, laminin incorporated on the inner surface of conduits can't be fabricated by conventional chemical method. In addition, the use of an oxygen plasma treatment can avoid the problems encountered in wet chemical method, such as residual solvent on the surface and the swelling of the substrates.

To display the changes in the microstructures of the Rhodamin-incorporated NCs following oxygen plasma treatment, the fluorescence and phase contrast images are shown in Fig. 1B. The longer the plasma treatment time is, the greater the damage of the chitosan structure is. For preserving the microstructure of chitosan scaffold and effectively increasing the amount of laminin incorporated, the most suitable treatment time is $5 \mathrm{~min}$. According to the outcome, the protein will penetrate the entire scaffold for $10 \mathrm{~min}$ treatment. The results showed that the unsuitable prolonging of treatment time would lead to the loss of the micro-architecture of the chitosan scaffold.

\section{Behavior analysis}

Fig. 2 shows the mean BBB and CBS open-field walking scores for the $\mathrm{NC}$ and $\mathrm{LN}-\mathrm{NC}$ experimental groups. According to the movie recording of the open-field locomotion of the representative animals, the injured hind limbs have the potential for recovery by both LN-NC and $\mathrm{NC}$ intervention. Although the BBB scores of both groups did not show significant functional recovery, the CBS scores achieved a mean of approximately 80 , which indicates the onset of behavior improvement. Compared with $\mathrm{NC}$ group, the experimental groups that received LN-NC transplantations achieved better results. Further- 

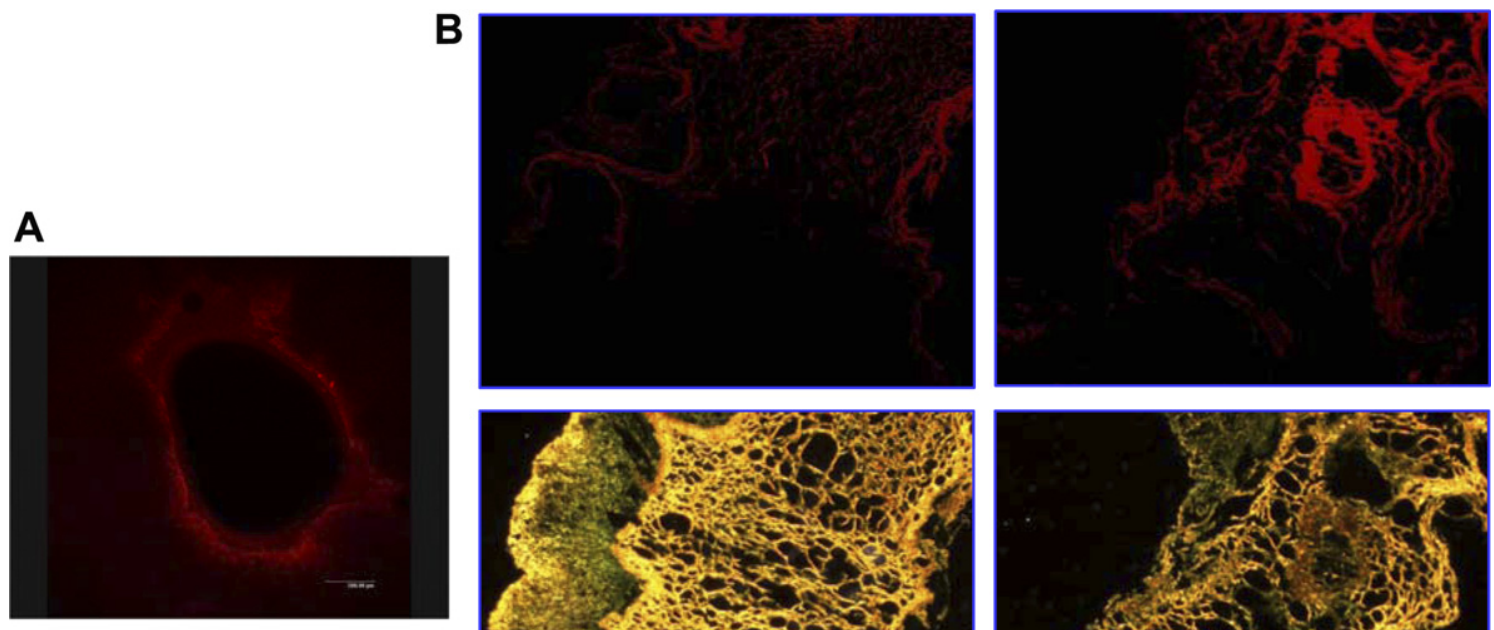

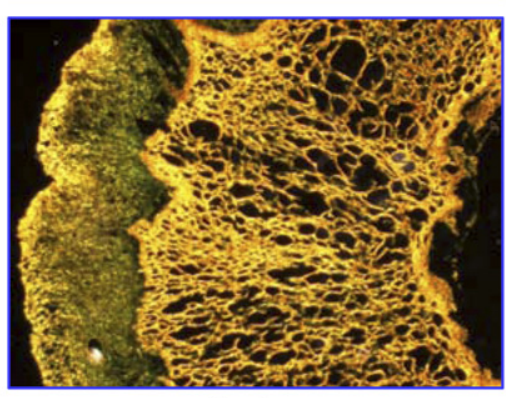

5 minutes

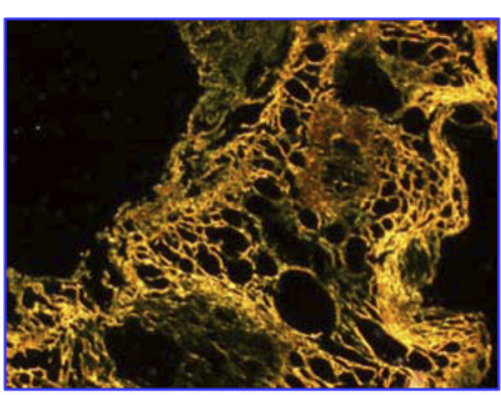

10 minutes

Fig. 1. Transverse sections of the Rhodamine-BSA-incorporated NCs. (A) The whole conduit with plasma treatment for 5 min. (B) Part of the transverse section reveals the microstructure of chitosan conduit with different plasma treatment time. Upper: fluorescence images; lower: phase contrast images.
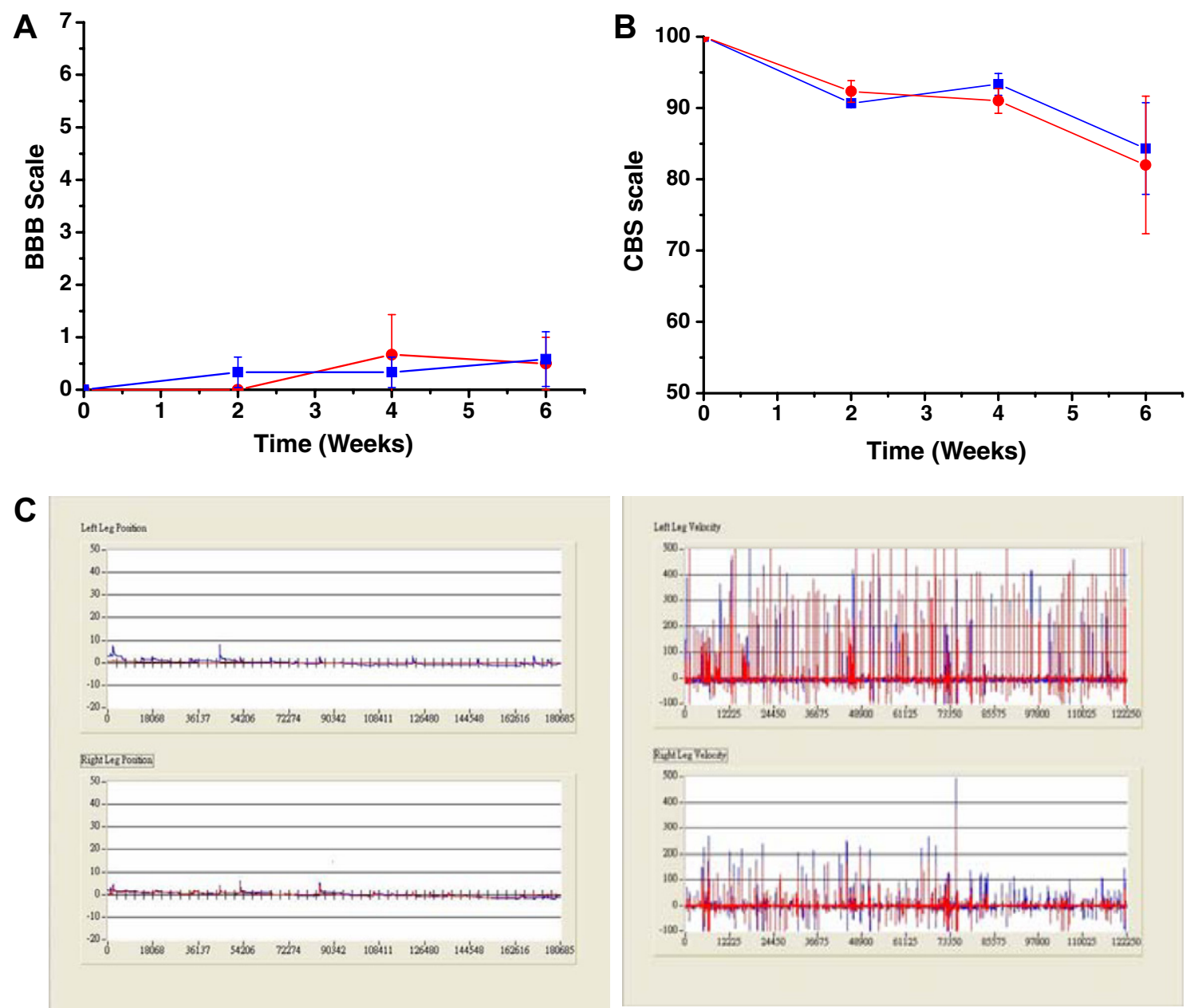

Fig. 2. Behavior analyses: (A) BBB scores (B) CBS scores. Red line: LN-NC groups; blue line: NC groups. (Data shown as means \pm standard deviations, $n=3$.) (C) Treadmill analysis of the NC mediated animals. Left: SCI just happened; right: two months post-injury. (For interpretation of color mentioned in this figure the reader is referred to the web version of the article.) 


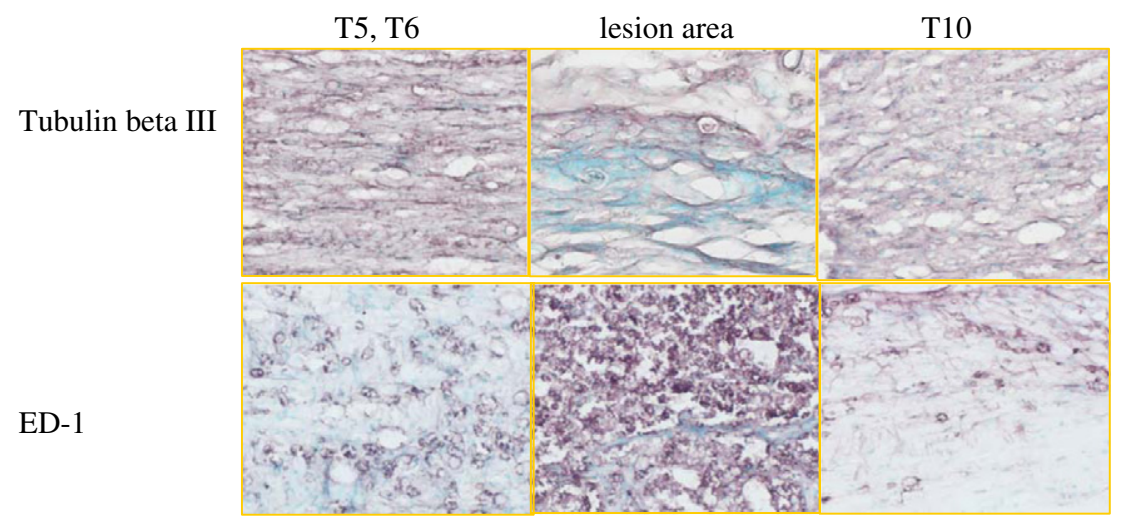

Fig. 3. Sections with different immunostained markers of LN-NC group at the injury epicenter at 30 days post-injury.

more, the outcome of treadmill analysis (Fig. 2C) at 2 months post-injury could be indicative of plantar-stepping improvement. Nonetheless, it is difficult to ascertain whether the stepping improvement proofed by treadmill analysis was the result of improvement in muscular or neural function. Therefore, what we got from BBB, CBS and treadmill analyses can only point to a tendency of behavior improvement. For this reason, immunohistochemistry and histological analyses need not only to follow, but also to precede, any future experiments.

\section{Immunohistochemistry}

Histology and immunocytochemistry were performed on all the cord tissue specimens to help elucidate the rats' functional recovery. The images in Fig. 3 were obtained from the animals whose CBS scores placed them near the mean of their respective groups. The large number of Tubulin beta III-positive cells found in the lesioned area indicates a large number of cells found. Furthermore, the ED-1 positive stain showed that macrophages had infiltrated the injury site in order to remove inhibitory debris, such as myelin, cellular debris, astrocytes, oligodendrocytes, and microglia. Macrophages can also produce cytokines, which enhance axonal re-grow. To obtain direct proof of axonal regeneration, we tested for the presence of GAP-43, a protein which is up-regulated in the growth cones of regenerating axons [24]. The presence of GAP43 was confirmed by the use of anti-GAP- 43 as primary antibody, and than analyzed by western blot. The related optic densities of GAP-43 positive bands (Supplementary Fig. 2) suggest a profile consistent with the occurrence of axonal regeneration. Compared with un-coated $\mathrm{NC}$ group, we detected a larger amount of GAP-43 in the LN-NC group. This suggests that LN-NC group have better efficiency for nerve regeneration.

In order to make sure that nerve regeneration following traumatic SCI was mediated by nerve conduits, we

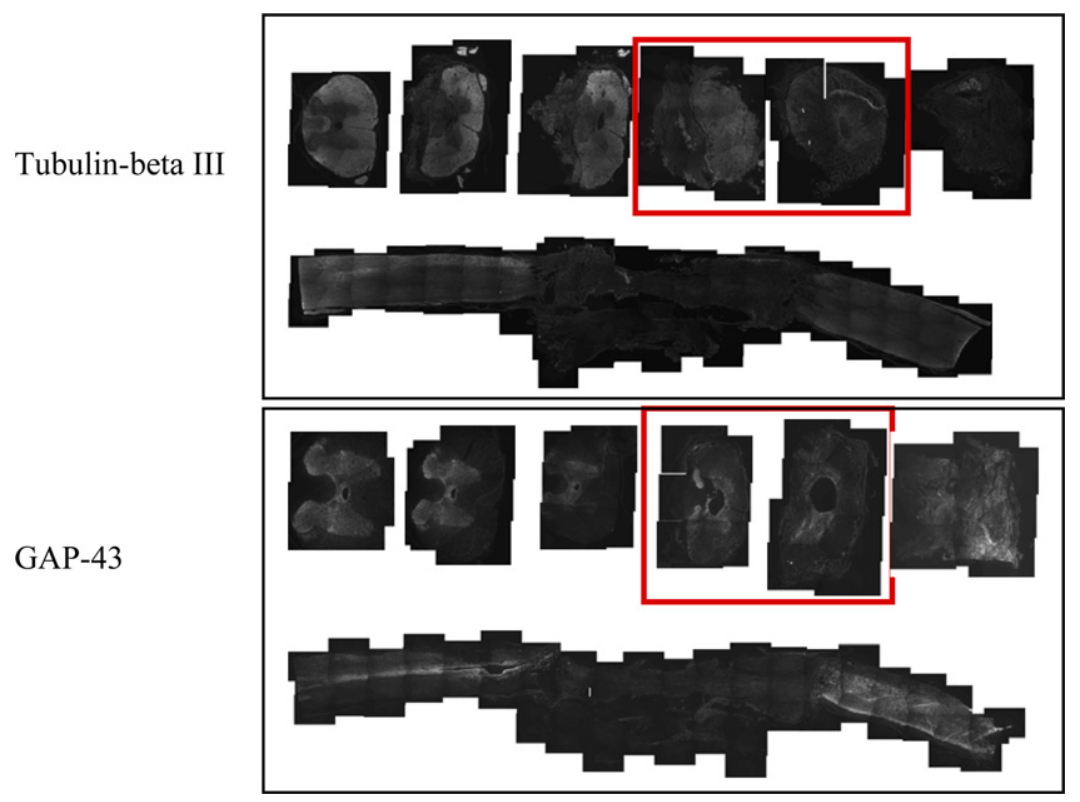

Fig. 4. Cross sections (upper) and longitudinal sections (lower) are got from NC group at 60 days post-injury. The images of the red frame are at the level of the lesion. 
obtained cross sections of spinal cord from T5 to T10, along with longitudinal sections along the midline of the spinal cord through the injury epicenter (Fig. 4). A large number of Tubulin-positive elements found to be distributed throughout the channel and scaffold further suggest a possible additional mechanism for cellular growth. In addition to the channel of the conduit, the porous chitosan scaffold also has good affinity for cell attachment. The positive GAP-43 results showed that most of the GAP-43 is distributed around the channel at 60 days post-injury. Furthermore, the nerve conduits exhibited immuno-negative staining for antibodies against both COX-2 and caspase-3 (data not shown). The negative results showed that no apoptosis occurred during nerve regeneration.

The result of GAP-43 staining that most of the axons went around the channel instead of growing through it could be explained by two assumptions. One is that the re-growing axons went through the channel first, and then attached to the channel surface over the following 2 months. This assumption could be verified by obtaining more sample sections of the conduit at specific times, such as once a week, during the 2-month time frame. The other assumption is that the axons simply favor growing around the channel. The diameter of the channel $(570 \mu \mathrm{m})$ is large in comparison with the nerve cells. It might be difficult for cells to re-grow in such a large area without any support. No matter which assumption is, our experiment has shown that our channel is still a guide for axon growth. We hypothesize that providing larger surface areas, such as multichannel-nerve conduits with decreasing diameters for cell attachment, or putting fibers into the channel, might enhance axon re-growth. Furthermore, seeding schwann cells on the channel surface to guide axon extension could also prove to be more efficient than simple nerve conduits for axonal re-growth. The scaffold we designed might not be the most suitable one. The pattern of the scaffold needed for the regeneration of nerve tissue in the nervous system should continue to be optimized in the near future.

\section{Conclusion}

Using oxygen plasma, we have successfully incorporated laminin into the inner surface of a nerve conduit in order to guide the re-growth of damaged axons following traumatic spinal cord injury. We found that prolonging the plasma treatment destroyed the microstructure of the porous chitosan scaffold. In our animal model experiment, the results obtained from BBB, CBS and treadmill analyses following the transplantation of nerve conduits showed a tendency towards behavior improvement. The immunopositive stain of ED-1, Tubulin beta III, and GAP-43 indicated that the nerve conduits could lead the damaged axons to extend across the injured area and rejoin their other end. The immuno-negative stain for COX-2 and Caspase- 3 indicated that the nerve conduits may not necessarily trigger inflammation or apoptosis post-surgery. Western blot analysis revealed that the optic density of the GAP-43 of LN-NC group was better than that of the NC group. Laminin did indeed increase the efficiency of axon regrowth.

\section{Acknowledgment}

We thank Dr. Lee Shoei-Sheng at the Institute of Pharmacy, National Taiwan University, for the ${ }^{1} \mathrm{H}$ NMR analysis.

\section{Appendix A. Supplementary data}

Supplementary data associated with this article can be found, in the online version, at doi:10.1016/j.bbrc. 2007.04.049.

\section{References}

[1] J.K. Terzis, D.D. Sun, P.K. Thanos, History and basic science review: past, present, and future of nerve repair, J. Reconstr. Microsurg. 13 (1997) 215-225.

[2] C.A. Heath, G.E. Rutkowski, The development of bioartificial nerve grafts for peripheral-nerve regeneration, TIBTECH 16 (1998) 163-168.

[3] Y.C. Huang, C.C. Huang, Y.Y. Huang, Manufacture of porous polymer nerve conduits through a lyophilizing and wire-heating process, J. Biomed. Mater. Res. Part B: Applied Biomaterials 74 (2005) 659-664.

[4] H.P. Gong, Y.H. Zhong, J.C. Li, Y.D. Gong, N.M. Zhao, X.F. Zhang, Studies on nerve cell affinity of chitosan-derived materials, J. Biomed. Mater. Res. 52 (2000) 285-295.

[5] J.C. Li, Y.H. Zhong, Y.D. Gong, N.M. Zhao, X.F. Zhang, Chitosan conduits for peripheral nerve regeneration, Tsinghua. Sci. Technol. 4 (1999) 1515-1518.

[6] P. Dongxu, C. Xiaodong, M. Lijiang, H. Yuanjie, X. Wenshu, S. Ruihuan, Z. Ying, Study on nerve cell affinity of polymer hydrogel films, in: Presented at the Third Far-Eastern Symposium on Biomedical Materials, July 15-17 (1997) Chengdu, China.

[7] T. Freier, R. Montenegro, H.S. Koh, M.S. Shoichet, Chitin based tubes for tissue engineering in the nervous system, Biomaterials 26 (2005) 4432-4624.

[8] A. Domard, M. Domard, Chitosan: structure-properties relationship and biomedical applications, in: S. Dumitriu (Ed.), Polymeric Biomaterials, Marcel Dekker, New York, 2002, pp. 187-212.

[9] J. Yang, J.Z. Bei, S.G. Wang, Improving cell affinity of poly (D,Llactide) film modified by anhydrous ammonia plasma treatment, Polym. Adv. Technol. 13 (2002) 220-226.

[10] Y.Q. Wan, J. Yang, J.L. Yang, J.Z. Bei, S.G. Wang, Cell adhesion on gaseous plasma modified poly (1-lactide) surface under shear stress field, Biomaterials 24 (2003) 3757-3764.

[11] J. Yang, G.X. Shi, J.Z. Bei, S.G. Wang, Y.L. Cao, Q.X. Shang, G.H. Yang, W.J. Wang, Fabrication and surface modification of macroporous poly (1-lactic acid) and poly (1-lactic-co-glycolic acid) (70/30) cells scaffold for human skin fibroblast cells culture, J. Biomed. Mater. Res. 62 (2002) 438-446.

[12] J. Yang, J.Z. Bei, S.G. Wang, Enhanced cell affinity of poly (d,1lactide) by combining plasma treatment with collagen anchorage, Biomaterials 23 (2002) 2607-2614.

[13] Y.C. Huang, Y.Y. Huang, K.S. Chen, Surface modification and characterization of Chitosan or PLGA membrane with laminin by chemical and oxygen plasma treatment for neural regeneration, $\mathrm{J}$. Biomedical Materials Research: Part A (2007). Available online 2 March 2007.

[14] L. Vachoud, N. Zydowicz, A. Domard, Formation and characterization of a physical chitin gel, Carbohydr. Res. 320 (1997) 169-177.

[15] M. Lavertu, Z. Xia, A.N. Serreqi, M. Berrada, A. Rodrigues, D. Wang, M.D. Buschmann, A. Gupta, A validated ${ }^{1} \mathrm{H}$ NMR method 
for the determination of the degree of deacetylation of chitosan, J. Pharm. Biomed. Anal. 32 (2003) 1149-1158.

[16] D.M. Basso, M.S. Beattie, J.C. Bresnahan, A sensitive and reliable locomotor rating scale for open field testing in rats, J. Neurotrauma 12 (1995) 1-21.

[17] K. Gale, H. Kerasidis, J. Wrathall, Spinal cord contusion in the rat: behavioral analysis of functional neurologic impairment, Exp. Neurol. 88 (1985) 123-134.

[18] J.A. Nessler et al., IEEE Trans. Neural Syst. Rehabil. Eng. 13 (2005) 497-506.

[19] T. Freier, H.S. Koh, K. Kazazian, M.S. Shoichet, Controlling cell adhesion and degradation of chitosan films by N-acetylation, Biomaterials 26 (2006) 5872-5878.
[20] H.M. Geller, J.W. Fawcett, Building a bridge: engineering spinal cord repair, Exp. Neurol. 174 (2002) 125-136.

[21] P.K. Thanos, S. Okajima, J.K. Terzis, Ultrastructure and cellular biology of nerve regeneration, J. Reconstr. Microsurg. 14 (1998) 423 436.

[22] M.B. Bunge, Bridging areas of injury in the spinal cord, Neuroscientist 7 (2001) 325-339.

[23] C.M. Chan, T.M. Ko, Polymer surface modification by plasma and photons, Surf. Sci. Report. 24 (1996) 1-54.

[24] L.I. Benowitz, P.J. Apostolides, N. Perrone-Bizzozero, S.P. Finklestein, H. Zwiers, Anatomical distribution of the growth-associated protein GAP-43/B-50 in the adult rat brain, J. Neurosci. 8 (1988) 339 352. 